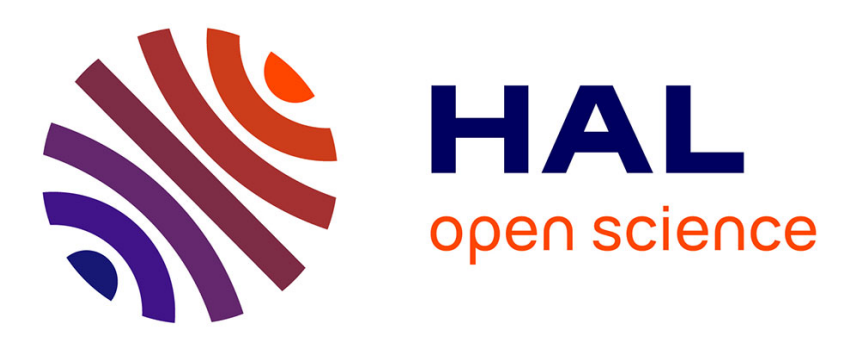

\title{
On the performance evaluation and analysis of general robots with mixed dofs
}

\author{
Samah Aref Shayya, Sébastien Krut, Olivier Company, Cédric Baradat, \\ François Pierrot
}

\section{- To cite this version:}

Samah Aref Shayya, Sébastien Krut, Olivier Company, Cédric Baradat, François Pierrot. On the performance evaluation and analysis of general robots with mixed dofs. IROS: Intelligent Robots and Systems, Sep 2014, Chicago, IL, United States. pp.490-497, 10.1109/IROS.2014.6942604 • lirmm01275349

\author{
HAL Id: lirmm-01275349 \\ https://hal-lirmm.ccsd.cnrs.fr/lirmm-01275349
}

Submitted on 1 Dec 2018

HAL is a multi-disciplinary open access archive for the deposit and dissemination of scientific research documents, whether they are published or not. The documents may come from teaching and research institutions in France or abroad, or from public or private research centers.
L'archive ouverte pluridisciplinaire HAL, est destinée au dépôt et à la diffusion de documents scientifiques de niveau recherche, publiés ou non, émanant des établissements d'enseignement et de recherche français ou étrangers, des laboratoires publics ou privés. 


\title{
On the Performance Evaluation and Analysis of General Robots with Mixed DoFs*
}

\author{
Samah Shayya ${ }^{(a, b)}$, Sébastien Krut ${ }^{(b)}$, Olivier Company ${ }^{(b)}$, Cédric Baradat ${ }^{(a)}$ and François Pierrot ${ }^{(b)}$
}

\begin{abstract}
This paper deals with the kinetostatic performance evaluation regarding translational and rotational motions of general robots with mixed operational degrees of freedom (dofs). Also, robots with different types of actuators can be analyzed based on the same approach without any problem. Besides, the generality of the approach embraces serial, parallel, actuatedly or kinematically redundant robots. The paper seeks clarifying the issue and providing a relevant solution. It also clarifies some other useful points in this matter.
\end{abstract}

\section{INTRODUCTION}

Performance evaluation of robots is among the most important and rich research fields in robotics. In fact, the research in this matter can be divided into two main directions (although they may intersect sometimes): the first is the establishment of innovative performance measures quantifying different aspects of robot's performance, and the second consists of finding relevant ways of tackling performance analysis of robots with mixed degrees of freedom (dofs) (case of non-homogeneous Jacobian matrices) on one hand and dealing properly with redundant robots (whether the redundancy is of actuation or kinematic type) on the other hand.

As a matter of fact, the abstract idea of the performance measure itself is not problematic and we always expect to have new performance measures evolving based on the industrial needs and tasks to be fulfilled by the mechanism to be designed. However, the problematic being dealt with in the second research direction is much more severe and critical, especially the branch dealing with mixed dofs. In fact, the problem with all the so far introduced performance measures is the need to carry some sort of operations on the Jacobian matrix. These operations necessitate having the Jacobian matrix homogeneous regarding physical units; but unfortunately, this is not always the case. Actually, a problem in homogeneity appears in the case where we have actuators of different types (i.e. rotational and prismatic), or when having the end-effector's dofs being mixed (i.e. rotational and translational dofs).

* This work has been supported partially by the French National Research Agency within the ARROW project (ANR 2011 BS3 006 01) and by Tecnalia France.

(a)-Tecnalia France - MIBI Building, 672 Rue du Mas de Verchant, $34000 \quad$ Montpellier, France. E-mails: samah.shayya/cedric.baradat@tecnalia.com.

(b)-Montpellier Laboratory of Informatics, Robotics, and Microelectronics (LIRMM in French), a cross-faculty research entity of the Montpellier University of Sciences and Technologies (UM2) and the French National Center for Scientific Research (CNRS), 161 rue Ada, $34095 \quad$ Montpellier, France. E-mails: shayya/krut/company/francois.pierrot@lirmm.fr.
Exemplification on performance measures are the condition number [1], manipulability [2], minimum singular value [3], motion/force transmissibility indices [4-6], largest inscribed ellipsoids semi-axes lengths based indices [7-8], force-velocity isotropy index introduced in [9-10], etc...Among the aforementioned indices, [4,7,8,9] for instance, have been developed to address the limitation and loss of significance of classical measures such as condition number, manipulability, minimum singular value (and all measures based on singular values), etc. in the case of redundancy. Also among the other works that addressed this issue is [11] in which the choice of appropriate Riemannian metric has been discussed.

As for the homogeneity issue, it is has been addressed in numerous works. In [12], the homogenization of the Jacobian matrix has been achieved via considering the velocities of two points of the mobile frame. Based on such reasoning, [13] developed a homogeneous Jacobian matrix by relating the velocities of three non-collinear points of the mobile frame to the actuators velocities. Also, [14] theoretically extended the idea of [13] and applied the same technique for the study of force-related performance. Note that motion/force transmissibility indices [4-6] do not present homogeneity problem and can also be considered as an approach to overcome this issue. Other works such as [15, 16] have addressed the problem based on the apparent power concept of the mechanism resulting in a homogeneous formulation of the problem regardless of having the actuators of different types or the operational dofs being mixed (rotational and translational); in fact, this has been achieved by introducing some weighting factors $K_{L}$ and $K_{R}$ (the ratio of which is important) and then carrying out a minimization of some quantity to get a unique value $\beta=K_{L} / K_{R}$ upon which the final power manipulabity depends. However, the relation of the established measures to operational velocities and forces is rather vague. This is not to mention the associated complexity. Moreover, one of the other approaches that have been suggested to deal with mixed dofs is considering the translational and rotational parts of the Jacobian matrix separately as highlighted in [17] to study manipulability. Similar approach, called direction selective indices (DSIs) [18], has been developed in which translational motion itself has been decomposed into its individual dofs and studied separately (based on manipulability index); such a method can similarly be applied for rotational dofs robots or robots with mixed dofs. It is also important to mention that among other spread methods to deal with homogenization is the use of some sort of weighting matrix or characteristic length such as [19, 20]. Unfortunately, all the aforementioned homogenization 
techniques are characterized by arbitrariness, and/or lack of direct comprehensible significance from kinetostatics point of view...

In this article, we provide a general framework and a relevant approach to assess kinetostatic performance of general robot with mixed dofs based on physically significant and well-understood measures; these measures are not characterized by arbitrariness as they do not require homogenization and also cope up well with redundant robots (whether actuatedly or kinematically redundant). Moreover, the measures to be presented are well-suited in the case of having actuators with different nature (i.e. in the case of having rotational and prismatic actuators at the same time).

The paper will provide the general approach at beginning of section (II) with the necessary details in the dedicated four subsections ((II.A) through (II.D)). The subsections of (II) decompose the issue into singularity measurement (subsection II.A), relevant kinetostatic and precision measurements (II.B and II.C), and essential remarks regarding redundancy (subsection II.D). Afterwards, section (III) presents a case study on the actuatedly redundant 3 dofs $(2 \mathrm{~T}-1 \mathrm{R})^{1}$ manipulator, DUAL V [22], as to illustrate the methodology. The paper ends in section (IV) with conclusions and perspectives.

\section{GENERAL APPROACH}

To address the problem correctly, we need to recall the simple case of performance evaluation; i.e. the case where the goal is performance evaluation of non-redundant robot that does not present any type of the homogeneity problems (all actuators are of same type, and all dofs are either translational or rotational). The condition number, manipulability, or singular values are beneficial in this case, as they give us measure of singularity (closeness of a pose to singularity) and kinetostatic performance significance regarding the velocity and force transmission values (isotropy of the transformation in velocity or force). Also, if we estimate joint space velocity capabilities (or force capabilities) to be described or estimated by unitary sphere, then the singular values and the condition number can serve as measure of isotropy of output performance in velocity and force (for more on that refer to [7-8]). Moreover, the norm of the velocity or force vector in this case is of solid significance.

What about robots with homogeneity problem? Now suppose that we have non-homogeneous Jacobian relative to the physical units. Then, whatever the homogenization technique (i.e. for example using weighting matrices [19, 20] or using two or three points on the mobile frame [12-14]), there is no direct comprehensible physical significance (according to our knowledge) of the norm of such homogenized velocity or homogenized force vector. Thus, the significance remaining for the condition number and other singular values based indices reduces to singularity measure which is also not unique due to the undoubted arbitrariness of the homogenization.

Therefore, a relevant way to deal with mixed dofs robots is as follows:

1. First study the singularities of the robot analytically or numerically to extract the exact or approximate values of the singular poses. These singularities include the classical series and parallel type singularities (inputoutput singularities) as well as constraint singularities. Then, based on the priori knowledge of these singular poses, one can choose any singular measure (condition number, manipulability, etc...) with any homogenization technique to assess singularity related performance. This will lead to setting the separation boundaries from singularities (this can be easily done by looking on how the singularity measure varies in the singular pose vicinity and as it goes away from singularity). In case of complex robots where we might have constraint singularities, the formulation of Jacobians must for sure consider passive joints as well. The arbitrariness here is still inevitable but not that critical.

2. Then, assess the kinetostatic performance separately for translational and rotational motions. Here only inputoutput relationships are concerned (i.e. Jacobians relating actuators' speeds (or torques) to operational twist (or wrench) are considered. However, the case of studying translational motion or rotational motion alone should be done with care. This is due to the fact that we are in a case similar to actuation redundancy where classical indices are no more as significant from kinetostatics point of view. It is important to note that still here if the actuators are not of the same type there is a homogeneity problem; but we will see that our approach to the problem is by no means arbitrary, because actuators types do not matter. Also, note that this step cannot replace the first one as it is not capable of detecting singularities, but only serves for kinetostatic analysis.

3. The optimization of a robot design can be established based on the kinetostatic analysis carried on translational and rotational motions separately considering the results of the first step (the singularities). In this case, if we are interested in both performances (translational and rotational), we will surely have a multi-objective optimization problem.

As we have generally introduced our approach to the problem at hand, it is time to give a relevant solution for kinetostatic analysis.... The global solution will be discussed in three subsections under the following guidelines:

1. Singularity Measure;

2. Isotropic Speeds and Isotropic Forces;

3. Precision Measures: Operational Resolutions.

A final subsection will emphasize some important remarks in the case of redundancy. 


\section{A. Singularity Measure}

As previously stated, we can utilize any of the classically used indices to assess singularity performance such as the condition number on the homogenized Jacobian matrix. But it might be good to suggest here more suited measures.

Consider now the general kinematic and force relations of any robot being given by:

$$
\dot{q}=J_{m} t \Leftrightarrow t=J \dot{q}
$$

and

$$
\boldsymbol{f}=\boldsymbol{J}_{\boldsymbol{m}}^{\mathrm{T}} \boldsymbol{\tau} \Leftrightarrow \boldsymbol{\tau}=\boldsymbol{J}^{\mathrm{T}} \boldsymbol{f}
$$

where $\dot{\boldsymbol{q}}$ and $\boldsymbol{t}=\left(\begin{array}{ll}\boldsymbol{v}^{\mathrm{T}} & \boldsymbol{\omega}^{\mathrm{T}}\end{array}\right)^{\mathrm{T}}$ are the joint velocities vector and the operational twist vector (linear $v$ and angular $\boldsymbol{\omega}$ velocities of the platform) respectively, and where $\boldsymbol{\tau}$ and $\boldsymbol{f}$ are the joint torques vector and the operational wrench (operational force and moment) respectively. As for $\boldsymbol{J}_{\boldsymbol{m}}$ and $\boldsymbol{J}$, they are the respective representation of the inverse and direct Jacobian matrices. The matrices $\boldsymbol{J}_{\boldsymbol{m}}$ and $\boldsymbol{J}$ are related to each other by matrix inversion procedure when they are square matrices (non-redundant) and by pseudoinversion in case of redundant robots (actuatedly redundant or kinematically redundant). The expressions of (1) and (2) are very famous and are given at regular non-singular pose. In fact, the two equivalent expressions of each equation are admitted without any problem in case of non-redundant robots; however, when we have actuation or kinematic redundancy, only one of the two forms of (1) and (2) is admitted. More precisely, in case of actuation redundancy only relations based on $\boldsymbol{J}_{\boldsymbol{m}}$ are naturally available as those based on $\boldsymbol{J}$ (pseudo-inverse of $\boldsymbol{J}_{\boldsymbol{m}}$ in this case) require considering some assumptions. As for kinematic redundancy, expressions of (1) and (2) that are based on $\boldsymbol{J}$ are naturally available, while those based on $\boldsymbol{J}_{\boldsymbol{m}}$ (pseudoinverse of $\boldsymbol{J}$ ) necessitate some assumptions. These aforementioned notes will be more discussed in separate subsection as not to cut the main idea's flow and maintain simplicity for now. So, as we seek simplicity, we will consider the case of non-redundant robot with 6 dofs (3T3R) (i.e. $\operatorname{dim}\left(\boldsymbol{J}_{\boldsymbol{m}}\right)=\operatorname{dim}(\boldsymbol{J})=6 \times 6$ with $\left.\boldsymbol{J}=\boldsymbol{J}_{\boldsymbol{m}}^{-1}\right)$. Also, we will assume that the actuators are of same type. Consider now the homogenized matrices $\boldsymbol{J}_{\boldsymbol{m} w}$ and $\boldsymbol{J}_{\boldsymbol{w}}$ derived from $\boldsymbol{J}_{\boldsymbol{m}}$ and $\boldsymbol{J}$ respectively, by using the characteristic length technique for example, i.e.:

$$
\left\{\begin{array}{l}
\boldsymbol{J}_{\boldsymbol{m} w}=\boldsymbol{J}_{\boldsymbol{m}} \boldsymbol{W}^{-1} \\
\boldsymbol{J}_{\boldsymbol{w}}=\boldsymbol{J}_{\boldsymbol{m} w}^{-1}=\boldsymbol{W} \boldsymbol{J} \\
\boldsymbol{W}=\operatorname{diag}\left(1,1,1, C L_{1}, C L_{2}, C L_{3}\right) \\
\text { with } C L_{i} \text { a charcteristic length }
\end{array}\right.
$$

Note that if we have different types of actuators, we should use another weighting matrix; but it is not the case here (for simplicity only without loss of generality).

Then, as a singularity measure one can use for example the condition number $\kappa$ defined by:

$$
\kappa=\operatorname{cond}\left(\boldsymbol{J}_{w}\right)=\operatorname{cond}\left(\boldsymbol{J}_{\boldsymbol{m} \boldsymbol{w}}\right)=\frac{\sigma_{\max }}{\sigma_{\min }}=\frac{\sigma_{\max }^{m}}{\sigma_{\min }^{m}}
$$

where $\sigma_{\min }$, and $\sigma_{\max }$ are minimum and maximum singular values of $\boldsymbol{J}_{\boldsymbol{w}}$ which are the inverse values of maximal and minimal singular values of $\boldsymbol{J}_{\boldsymbol{m} \boldsymbol{w}}$ denoted by $\sigma_{\max }^{m}$ and $\sigma_{\min }^{m}$.

But the condition number $\kappa$ or its reciprocal $1 / \kappa$ are tricky in the situation where both minimal and maximal singular values go toward very high value $\left(\sigma_{\max }=\sigma_{\min } \rightarrow \infty\right)$ or very small value $\left(\sigma_{\max }=\sigma_{\min } \rightarrow 0\right)$ simultaneously resulting in a value of unity (indicating a very good situation); however, we are near singularity. So, to overcome this drawback of condition number, we would suggest a better suited index based on singular values which is the $M S$ index (measure of singularity index) defined by:

$$
M S=\min \left(\sigma_{\min }, \frac{1}{\sigma_{\max }}\right)=\min \left(\frac{1}{\sigma_{\max }^{m}}, \sigma_{\min }^{m}\right)
$$

Note that $M S \leq 1$ and it is best when closer to unity value. Obviously, $M S$ does not skip any singularity case and hence more suited than the condition number in all cases. It is worth mentioning that this index has been implicitly used by [21] for the optimization of Orthoglide. Note that here we are speaking in case of simple robot where singularities can be of input-output type; otherwise in the case where we might have constraint singularities, one must perform the analysis including the passive joints as well in the Jacobians.

Another possible singularity measure can be the FVI (Force-Velocity Isotropy) index suggested and used in [910] and given by:

$$
F V I=\min \left(\frac{v_{i s o}}{v_{w l}}, \frac{f_{i s o}}{f_{w l}}\right)
$$

with $v_{w l}$ and $f_{w l}$ being the lower bounds desired for isotropic homogenized speed and isotropic homogenized static force respectively (the two values are set by the designer or end-user). As for $v_{i s o}$ and $f_{\text {iso }}$, these are the isotropic homogenized speed and the isotropic homogenized static force, respectively (i.e. radii of largest spheres included in their corresponding operational zonotopes). This means the robot can move at least at a homogenized speed $v_{i s o}$ in all directions and can support at least a homogenized static force $f_{\text {iso }}$ regardless of direction. For sure and as previously demonstrated, such homogenized values are less indicative regarding kinetostatic performance and only serve as measure of singularity (as any other performance measure): a good performance regarding singularity is when $F V I$ is high and worst performance is when $F V I$ is near zero ( $v_{i s o} \rightarrow 0$ implies series-type singularity, and $f_{\text {iso }} \rightarrow 0$ implies parallel-type singularity). Note that a value of $F V I \geq 1$ indicates that the desired limits are satisfied. The calculation of $v_{\text {iso }}$ and $f_{\text {iso }}$ is quite simple as follows: 


$$
\left\{\begin{array}{l}
v_{\text {iso }}=\min _{i=1 \ldots m}\left(\frac{\dot{q}_{\max _{i}}}{\left\|\dot{j}_{\boldsymbol{m w r}_{i}}\right\|}\right) \\
f_{\text {iso }}=\min _{i=1 \ldots m}\left(\frac{\tau_{\max _{i}}}{\left\|\boldsymbol{j}_{w_{i}}\right\|}\right) \\
m: \text { number of actuators }
\end{array}\right.
$$

where $\dot{q}_{\max _{i}}$ and $\tau_{\max _{i}}$ are respectively the maximal attainable speed and maximal torque or force capacity of the i-th actuator. As for $\boldsymbol{j}_{\boldsymbol{m} w \boldsymbol{r}_{i}}$ and $\boldsymbol{j}_{w c_{i}}$, they represent respectively the $\mathrm{i}$-th row vector and $\mathrm{i}$-th column vector of $\boldsymbol{J}_{\boldsymbol{m} w}$ and $\boldsymbol{J}_{w}$. Note that $\|$ vector $\|$ stands for the Euclidean norm of vector vector and this holds throughout the paper. It is worth mentioning that in the case of FVI index, homogenization relative to operational dofs is needed but no homogenization is required regarding actuators' types (in case of actuators of different nature). Thus, FVI is uniquely formulated regarding mixed actuators (because one is obliged to use the actuators' maximal speeds and maximal torques only) as compared to other classical indices such as condition number, manipulability, $M S$ etc...

Based on the latter argument, we recommend the use of $F V I$ as a singularity measure for any robot. For FVI index, robots with mixed types of actuators but same nature of dofs do not require any homogenization or special treatment. In such a case, the kinetostatic significance regarding isotropic speed and isotropic force capabilities is retained.

\section{B. Isotropic Speeds and Isotropic Forces}

In the previous part, we have introduced the FVI index that only loses its kinetostatic significance regarding velocity and force in the case of mixed dofs robots. Now, we will provide the modified version of $F V I$ to study kinetostatic performance of mixed dofs robots. In this case, we are not concerned except with input-output relationships between joint velocities (or joints' torques) and operational twist (or operational wrench i.e. force and moment, respectively); as here only kinetostatic performance is sought and such study cannot replace the explicit prior singularity analysis as early mentioned.

Let us consider the translational (or positional) parts of $\boldsymbol{J}_{\boldsymbol{m}}$ and $\boldsymbol{J}$ being respectively $\boldsymbol{J}_{\boldsymbol{m} p}$ and $\boldsymbol{J}_{p}$. Also, let the orientation or rotational parts of $\boldsymbol{J}_{\boldsymbol{m}}$ and $\boldsymbol{J}$ be respectively $\boldsymbol{J}_{\boldsymbol{m} \boldsymbol{o}}$ and $\boldsymbol{J}_{\boldsymbol{o}}$. This means, we have $\boldsymbol{J}_{\boldsymbol{m}}=\left[\begin{array}{ll}\boldsymbol{J}_{\boldsymbol{m} \boldsymbol{p}} & \boldsymbol{J}_{\boldsymbol{m} \boldsymbol{o}}\end{array}\right]$ and $\boldsymbol{J}=\left[\begin{array}{ll}\boldsymbol{J}_{\boldsymbol{p}}^{\mathrm{T}} & \boldsymbol{J}_{\boldsymbol{o}}^{\mathrm{T}}\end{array}\right]^{\mathrm{T}} . \quad$ Recall that $\boldsymbol{t}=\left(\begin{array}{ll}\boldsymbol{v}^{\mathrm{T}} & \boldsymbol{\omega}^{\mathrm{T}}\end{array}\right)^{\mathrm{T}} \quad$ and $\boldsymbol{f}=\left(\begin{array}{ll}\boldsymbol{f}_{\boldsymbol{p}}^{\mathrm{T}} & \boldsymbol{m}_{\boldsymbol{o}}^{\mathrm{T}}\end{array}\right)^{\mathrm{T}}$ where $\boldsymbol{f}_{\boldsymbol{p}}$ and $\boldsymbol{m}_{\boldsymbol{o}}$ are the force and moment applied on the platform.

Then, the isotropic linear speed $v_{\text {piso }}$ (in the case $\boldsymbol{\omega}=\mathbf{0}$ ) and isotropic pure static force $f_{\text {piso }}$ (in the case $\boldsymbol{m}_{\boldsymbol{o}}=\mathbf{0}$ ) are given by:

$$
\left\{\begin{array}{l}
v_{\text {piso }}=\min _{i=1 \ldots m}\left(\frac{\dot{q}_{\max _{i}}}{\left\|\dot{j}_{m p r_{i}}\right\|}\right) \\
f_{\text {piso }}=\min _{i=1 \ldots m}\left(\frac{\tau_{\max _{i}}}{\left\|\boldsymbol{j}_{p c_{i}}\right\|}\right) \\
m: \text { number of actuators }
\end{array}\right.
$$

with $\boldsymbol{j}_{\boldsymbol{m} \boldsymbol{r} \boldsymbol{r}_{i}}$ and $\boldsymbol{j}_{\boldsymbol{p} \boldsymbol{c}_{\boldsymbol{i}}}$ being the i-th row vector of $\boldsymbol{J}_{\boldsymbol{m} \boldsymbol{p}}$ and i-th column vector of $\boldsymbol{J}_{p}$ respectively. As a result, the translational $F V I$ index denoted by $F V I_{P}$ is given for $\boldsymbol{\omega}=\boldsymbol{m}_{o}=\mathbf{0}$ by:

$$
F V I_{p}=\min \left(\frac{v_{p i s o}}{v_{p w l}}, \frac{f_{p i s o}}{f_{p w l}}\right)
$$

where $v_{p w l}$ and $f_{p w l}$ are the lower limits desired for $v_{p i s o}$ and $f_{\text {piso }}$ respectively.

Similarly, we can calculate the isotropic angular speed $\omega_{i s o}$ (case $\boldsymbol{v}=\mathbf{0}$ ) and isotropic static torque or moment $m_{i s o}$ (case $\boldsymbol{f}_{p}=\mathbf{0}$ ) as follows:

$$
\left\{\begin{array}{l}
\omega_{i s o}=\min _{i=1 \ldots m}\left(\frac{\dot{q}_{\text {max }_{i}}}{\left\|\dot{j}_{\text {mor }_{i}}\right\|}\right) \\
m_{i s o}=\min _{i=1 \ldots m}\left(\frac{\tau_{\text {max }_{i}}}{\left\|\boldsymbol{j}_{\boldsymbol{o c}_{i}}\right\|}\right) \\
m: \text { number of actuators }
\end{array}\right.
$$

where $\boldsymbol{j}_{\boldsymbol{m o r}_{i}}$ and $\boldsymbol{j}_{\boldsymbol{o c}}$ being the i-th row vector of $\boldsymbol{J}_{\boldsymbol{m} \boldsymbol{i}}$ and ith column vector of $\boldsymbol{J}_{\boldsymbol{o}}$ respectively. Hence, the rotational motion $F V I$ index denoted by $F V I_{o}$ is given for $\boldsymbol{v}=\boldsymbol{f}_{\boldsymbol{p}}=\mathbf{0}$ by:

$$
F V I_{o}=\min \left(\frac{\omega_{i s o}}{\omega_{w l}}, \frac{m_{i s o}}{m_{w l}}\right)
$$

The terms $\omega_{w l}$ and $m_{w l}$ are the corresponding desired lower bounds on $\omega_{i s o}$ and $m_{i s o}$.

Note that here we considered translational motion capabilities in velocity and force for angular velocity being controlled to zero and having no external torques (and viceversa), but we can do the same study, for example fixing a certain angular velocity or fixing a certain applied external torque (and vice-versa) without any problem. Suppose for example, we need to know the isotropic linear speed $v_{i s o}^{s p}$ for the special case where the angular velocity is assumed to be $\boldsymbol{\omega}^{s p} \neq \mathbf{0}$, then we have:

$$
\dot{q}-J_{m o} \omega^{s p}=J_{m p} v
$$

Based on (12), we have:

$$
v_{i s o}^{s p}=\min _{i=1 \ldots m}\left(\frac{\dot{q}_{\max _{i}}^{\text {new }}}{\left\|\boldsymbol{j}_{\boldsymbol{m p r _ { i }}}\right\|}\right)
$$

with $\dot{q}_{\text {max }_{i}}^{\text {new }}=\min \left(\left|-\dot{q}_{\max _{i}}-\dot{q}_{i}^{\text {off }}\right|,\left|\dot{q}_{\max _{i}}-\dot{q}_{i}^{\text {off }}\right|\right)$ where $\dot{q}_{i}^{\text {off }}$ is the i-th component of $\dot{q}^{\text {off }}=J_{m o} \omega^{s p}$ and provided that 
$\left(-\dot{q}_{\max _{i}}-\dot{q}_{i}^{\text {off }}\right) \leq 0$ and $\left(\dot{q}_{\max _{i}}-\dot{q}_{i}^{\text {off }}\right) \geq 0$ for all $i=1 \ldots m ;$ otherwise $v_{i s o}^{s p}=0$ by convention as the robot initially cannot provide $\omega^{s p}$ angular velocity.

So far we have studied the kinetostatic performance of mixed dofs robots regarding isotropic speeds, and isotropic static loads in case of translation and in case of rotation. It is worth mentioning that in all FVI indices, the number of actuators $m$ can be greater or equal to number of dofs (i.e. applicable on redundant robots as well: for more clarification refer to the Remark subsection). In what follows, we will deal with the precision related measures of mixed dofs robots.

\section{Precision Measures: Operational Resolutions}

Among the significances of singular values-as applied in the case of non-redundant robot with homogeneous actuators and homogeneous dofs- is that the minimum and maximum singular values express how the joint errors are amplified in the operational space. In fact, we have:

$$
\delta q=J_{m} \delta x \Leftrightarrow \delta x=J \delta q
$$

where $\delta \boldsymbol{q}$ and $\delta \boldsymbol{x}$ are very small displacements in the joint and operational spaces respectively. Then, if $\sigma_{\min }$ and $\sigma_{\max }$ are the smallest and largest singular values of $\boldsymbol{J}$, one is interested in having $\sigma_{\max }$ as small as possible. In such a case, any error in the joint positions is not amplified in the operational space yielding a precise robot. However, this means reducing the velocity capabilities. A good compromise then would be to have $\sigma_{\min }$ and $\sigma_{\max }$ not only close to each other (meaning $\kappa=\operatorname{cond}(J)=1$ ), but rather having $\sigma_{\min }=\sigma_{\max }=1$ or $M S=1$; another reason why $M S$ is favored over $\kappa$ in such a case.

However, in case of robots with mixed dofs, singular values and based-upon indices are no more useful. So, to assess precision related performance of mixed dofs robot, one can directly study the translational and rotational operational resolutions.

Operational resolution is defined as the smallest step or increment the robot can do in the operational space and can be detected (by having the joint sensors increment by one count). In case of mixed dofs robots, there are two operational resolutions: translational and rotational ones.

We will give the mathematical formulation of the operational resolutions in the case of redundant and nonredundant robots.

Let us start with the case of actuation redundancy. In such a case, the inverse kinematics is unique (i.e. $\boldsymbol{J}_{m}$ is unique and available naturally), while the direct or forward kinematics $\boldsymbol{J}$ requires a pseudo-inversion procedure (with a synchronization condition to be fulfilled by joints' displacements and velocities). To clarify this, it would be good to recall the general closure or constraint equation (relating joint positions $\boldsymbol{q}$ and operational pose $\boldsymbol{x}$ ) of the form:

$$
\mathbf{F}(\boldsymbol{q}, \boldsymbol{x})=\mathbf{0}
$$

Then, differentiating (15) with respect to joint position vector $\boldsymbol{q}$ and operational pose $\boldsymbol{x}$, we get:

$$
\left\{\begin{array}{l}
J_{q} d q=J_{x} d x \\
J_{q}=\frac{\partial \mathbf{F}}{\partial \boldsymbol{q}}, J_{x}=-\frac{\partial \mathbf{F}}{\partial \boldsymbol{x}}
\end{array}\right.
$$

Thus, in case of actuation redundancy $\boldsymbol{J}_{\boldsymbol{x}}$ is non-square and $\boldsymbol{J}_{q}$ is a square matrix. This means we have naturally $\boldsymbol{J}_{\boldsymbol{m}}$ given by:

$$
\boldsymbol{J}_{\boldsymbol{m}}=\boldsymbol{J}_{q}^{-1} \boldsymbol{J}_{\boldsymbol{x}}
$$

The joint displacement in the case of actuation redundancy is therefore subject to synchronization condition described by:

$$
\left[\operatorname{null}\left(\boldsymbol{J}_{m}^{\mathrm{T}}\right)\right]^{\mathrm{T}} \boldsymbol{\delta} \boldsymbol{q}=\mathbf{0}
$$

meaning:

$$
\left\{\begin{array}{l}
\boldsymbol{\delta} \boldsymbol{x}=\boldsymbol{J} \boldsymbol{\delta} \boldsymbol{q} \\
\text { with : } \\
\boldsymbol{J}=\boldsymbol{J}_{\boldsymbol{m}}^{*} \text { : pseudo-inverse of } \boldsymbol{J}_{\boldsymbol{m}}
\end{array}\right.
$$

The non-redundant case is a special case of actuation redundancy, in which $\operatorname{null}\left(\boldsymbol{J}_{m}^{\mathrm{T}}\right)=\{\varnothing\}$ (at non-singular pose which is always assumed as we are studying performance at regular pose or in the vicinity of singularity but not at singularity).

Regarding kinematic redundancy, we have in this case $\boldsymbol{J}_{\boldsymbol{q}}$ non-square matrix, while $\boldsymbol{J}_{\boldsymbol{x}}$ is a square matrix. In this case, the Jacobian $\boldsymbol{J}$ is available and uniquely given by:

$$
\boldsymbol{J}=\boldsymbol{J}_{x}^{-1} \boldsymbol{J}_{q}
$$

In such a case, there is no constraint to be satisfied by the joints' displacements, and there is no unique inverse kinematic solution. That is to say:

$$
\left\{\begin{array}{l}
\boldsymbol{\delta} \boldsymbol{q}=\boldsymbol{J}_{m} \boldsymbol{\delta} \boldsymbol{x}+[\operatorname{null}(\boldsymbol{J})] \boldsymbol{\lambda} \\
\text { with } \lambda \in \mathbb{R}^{m-n} \text { (arbitrary vector) } \\
\boldsymbol{J}_{m}=\boldsymbol{J}^{*}: \text { pseudo-inverse of } \boldsymbol{J} \\
m: \text { no. of actuators \& } n: \text { no. of dofs }
\end{array}\right.
$$

Also, non-redundancy can be considered a special case of kinematic redundancy where $\operatorname{null}(\boldsymbol{J})=\{\varnothing\}$ (at regular pose).

After we have clarified some points regarding redundancy in its both types (actuation and kinematic), it is time to proceed with the calculation of translational and rotational resolutions of the robot. First, we define the feasible joint displacement zonotope $\boldsymbol{Z}_{q}$ as follows:

$$
\begin{aligned}
& \left\{\begin{array}{l}
\cdot \boldsymbol{Z}_{q}=\left\{\begin{array}{l}
\boldsymbol{\delta} \boldsymbol{q} \in \mathbb{R}^{m} ;\left|\delta q_{i}\right| \leq R_{q i} \forall i=1 \ldots m, \\
\text { and }\left[\operatorname{null}\left(\boldsymbol{J}_{m}^{\mathrm{T}}\right)\right]^{\mathrm{T}} \boldsymbol{\delta} \boldsymbol{q}=\mathbf{0}
\end{array}\right.
\end{array}\right\} \\
& \boldsymbol{Z}_{\boldsymbol{q}}=\left\{\boldsymbol{\delta} \boldsymbol{q} \in \mathbb{R}^{m} ;\left|\delta q_{i}\right| \leq R_{q i} \quad \forall i=1 \ldots m\right\} \\
& \text { (case of kinematic redundancy or non-redundancy) }
\end{aligned}
$$


where $R_{q i}$ is the resolution of the $\mathrm{i}$-th joint sensor.

Then, the translational operational resolution is defined as:

$$
\operatorname{Res}_{p}=\max _{\delta \boldsymbol{q} \in Z_{q}}\left(\left\|\boldsymbol{J}_{p} \delta \boldsymbol{q}\right\|\right)
$$

As for the rotational operational resolution, it is defined as:

$$
\operatorname{Res}_{o}=\max _{\delta \boldsymbol{q} \in Z_{q}}\left(\left\|\boldsymbol{J}_{o} \delta \boldsymbol{q}\right\|\right)
$$

It is quite important to highlight that although the expressions of the resolutions in (23) and (24) indicate an optimization procedure over $\boldsymbol{Z}_{q}$, yet these optimum solutions over the joint displacements' zonotope $Z_{q}$ can be computed analytically. The zonotope being convex with vertices and straight line edges and linearly mapped by $\boldsymbol{J}_{\boldsymbol{p}}$ or $\boldsymbol{J}_{\boldsymbol{o}}$ into the operational translational or rotational spaces, we know that the maximum will be at one of the $\boldsymbol{Z}_{q}$ vertices (also can be said at one of the vertices of the image of $Z_{q}$ by the linear mapping of $\boldsymbol{J}_{\boldsymbol{p}}$ or $\boldsymbol{J}_{\boldsymbol{o}}$ ). This simply means if we have $\boldsymbol{V}_{i}$ with $i=1 \ldots N$ ( $N$ being the number of vertices of $\boldsymbol{Z}_{q}$ ), we can rewrite (23) and (24) respectively as:

$$
\operatorname{Res}_{p}=\max _{i=1 \ldots N}\left(\left\|\boldsymbol{J}_{p} \boldsymbol{V}_{i}\right\|\right)
$$

and

$$
\operatorname{Res}_{o}=\max _{i=1 \ldots N}\left(\left\|\boldsymbol{J}_{o} \boldsymbol{V}_{i}\right\|\right)
$$

It is important to highlight that when we calculate $\operatorname{Res}_{p}$, we do not impose a condition on orientation or rotational displacement to be zero. Similarly, in the case of calculating $\operatorname{Res}_{o}$, we do not impose a condition on the translational displacement to be zero.

The robot is more precise when both resolutions are small and less precise as their values increase.

Note that the calculation methodology used here for operational resolutions can also be utilized to compute the theoretical translational and rotational repeatabilities and accuracies due to joints' repeatabilities and accuracies. This is done by replacing $\mathrm{i}$-th joint resolution $R_{q_{i}}$ by the corresponding joint position repeatability or accuracy.

Moreover and as a complement to this analysis, it is quite important to highlight some relations with peak linear speed and peak angular speeds. First, let us suppose for example that all actuators are identical, meaning $R_{q i}=R_{q} \forall i=1 \ldots m$ and $\dot{q}_{\max _{i}}=\dot{q}_{\max } \forall i=1 \ldots m$. Then, it is clear that the peak linear speed $v_{\text {peak }}$ and angular peak speed $\omega_{\text {peak }}$ satisfy:

$$
\left\{\begin{array}{l}
\frac{v_{\text {peak }}}{\operatorname{Res}_{p}}=\frac{\dot{q}_{\max }}{R_{q}} \\
\frac{\omega_{\text {peak }}}{\operatorname{Res}_{o}}=\frac{\dot{q}_{\max }}{R_{q}}
\end{array},\right. \text { all actuators being identical }
$$

(Note that to calculate peak linear speed or peak angular speed, we proceed in same manner as for the resolution, but replacing $R_{q i}$ by $\dot{q}_{\max }, \delta q_{i}$ by $\dot{q}_{i}$, and $\boldsymbol{\delta} \boldsymbol{q}$ by $\dot{\boldsymbol{q}}$. Also, note that when we calculate peak linear speed, we do not impose any condition on $\omega$ such as being zero i.e. $\omega$ can be any angular velocity. Similar explanation regarding peak angular speed.)

Thus peak operational speeds and resolutions are directly related, and their perfection is antagonist: meaning improving performance regarding resolution is accompanied by peak speed deterioration and vice-versa. This is generally the case.

Before ending this section, it is important to emphasize some remarks. These are given in the upcoming subsection.

\section{Remarks}

The FVI indices considering homogenized motion, translational motion and rotational motion have the same expressions in the case of actuation and kinematic redundancy as indicated in II.B, but some attention should be given regarding the meaning.

In actuation redundancy, the joint velocities should be synchronized obeying the condition $\left[\operatorname{null}\left(\boldsymbol{J}_{m}^{\mathrm{T}}\right)\right]^{\mathrm{T}} \dot{\boldsymbol{q}}=\mathbf{0}$, but the joint torques do not necessarily have to be perpendicular to the null space of $\boldsymbol{J}_{m}^{\mathrm{T}}$; however, the formulae given for isotropic force are based on the assumption of minimum norm solution of the torque i.e. $\left[\operatorname{null}\left(\boldsymbol{J}_{\boldsymbol{m}}^{\mathrm{T}}\right)\right]^{\mathrm{T}} \boldsymbol{\tau}=\mathbf{0}$ and which can be achieved via control means (note that if we are to consider isotropic force without the condition of orthogonality to null space, we will obtain a larger or equal value due to the fact that minimum norm solution set described by $\boldsymbol{\tau}=\boldsymbol{J}^{\mathrm{T}} \boldsymbol{f}$ is a subset of the generalized solution described by $\boldsymbol{\tau}=\boldsymbol{J}^{\mathrm{T}} \boldsymbol{f}+\left[\operatorname{null}\left(\boldsymbol{J}_{\boldsymbol{m}}^{\mathrm{T}}\right)\right]^{\mathrm{T}} \boldsymbol{\lambda} \quad$ with $\lambda \in \mathbb{R}^{m-n}$ for $m$ actuators and $n$ dofs, and where $\boldsymbol{J}$ is the pseudo-inverse of $\boldsymbol{J}_{\boldsymbol{m}}$ ).

As for kinematic redundancy, the situation between joint torques and joint velocities is interchanged. In this case, we have the Jacobian $\boldsymbol{J}$ unique, whereas for the inverse kinematics we have no unique solution and it is generally given by (21).

Then, the value of isotropic speed in this case and as given in the previous formulae (in section II.B) corresponds to the case of minimum norm solution of the inverse kinematics, i.e. for $\boldsymbol{\lambda}=\mathbf{0}$. Note that if we are to consider the general solution of (21) without considering the orthogonality condition to null space, we will obtain a larger or equal value for similar reason as indicated for isotropic force in case of actuation redundancy.

As for the forces study in case of kinematic redundancy, the joint torques must necessarily obey the following condition (orthogonality to null space of $\boldsymbol{J}$ ):

$$
[\operatorname{null}(\boldsymbol{J})]^{\mathrm{T}} \boldsymbol{\tau}=\mathbf{0}
$$

The formulae of isotropic forces in II.B are in agreement with the condition expressed in (28) (note that $\left.\operatorname{null}(\boldsymbol{J})=\operatorname{null}\left(\boldsymbol{J}_{\boldsymbol{m}}^{\mathrm{T}}\right)\right)$.

Thus, in all the cases the formulae given in section II.B are applicable without any problem in both cases of 
redundancy. Moreover, if other values for isotropic forces (case of actuation redundancy) exist, then these would be larger than those calculated by the aforementioned formulae. Similar conclusion can be derived regarding isotropic speeds in the case of kinematic redundancy.

\section{CASE STUdY: DUAL V}

To illustrate the suggested methodology, we provide a case study on the actuatedly redundant parallel manipulator, DUAL V [22], with planar motion (3 dofs, 2T-1R). The manipulator is depicted in Fig. 1 with the necessary

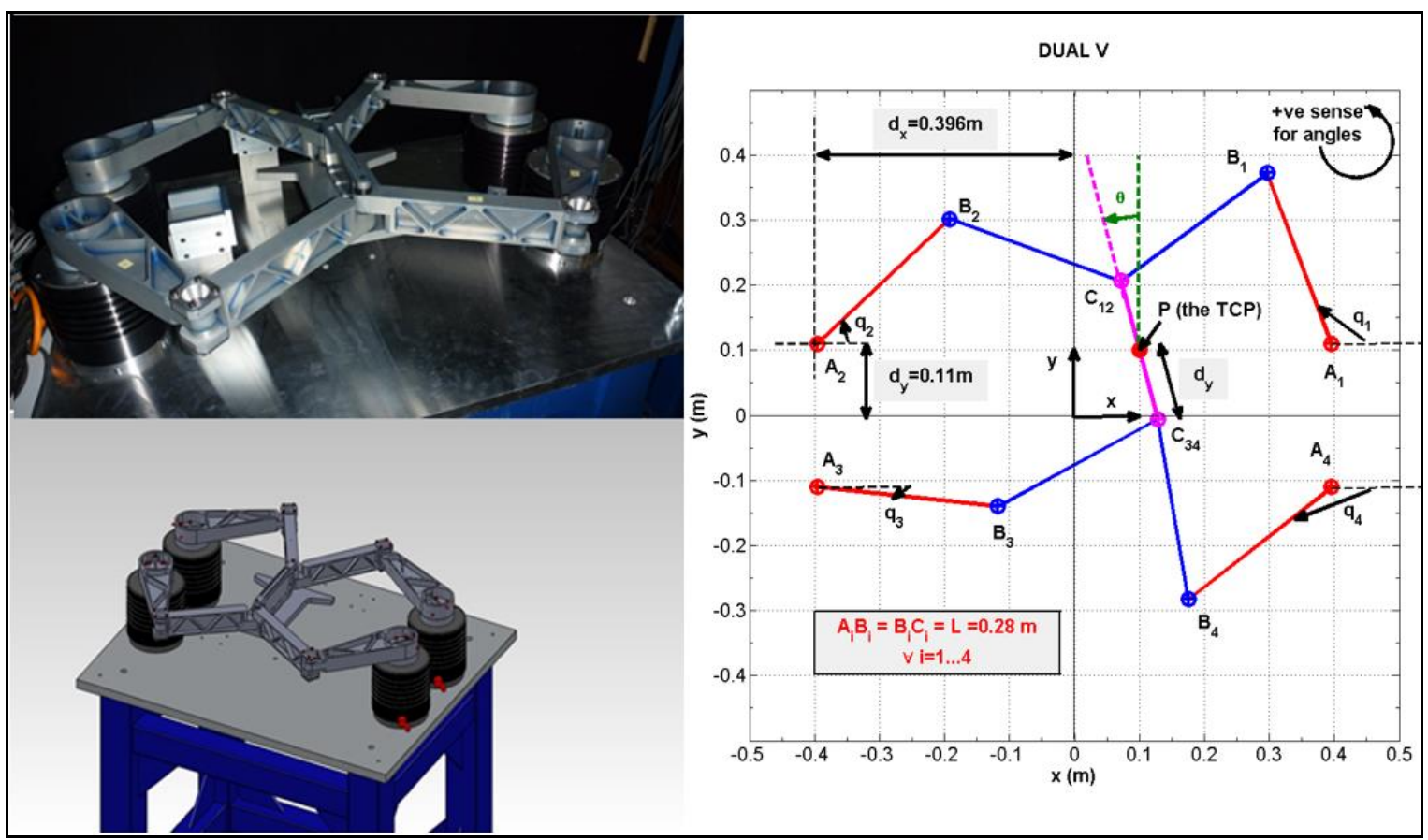

Figure 1. DUAL V Prototype, CAD drawing and simplified schematic diagram showing the principal geometric parameters.

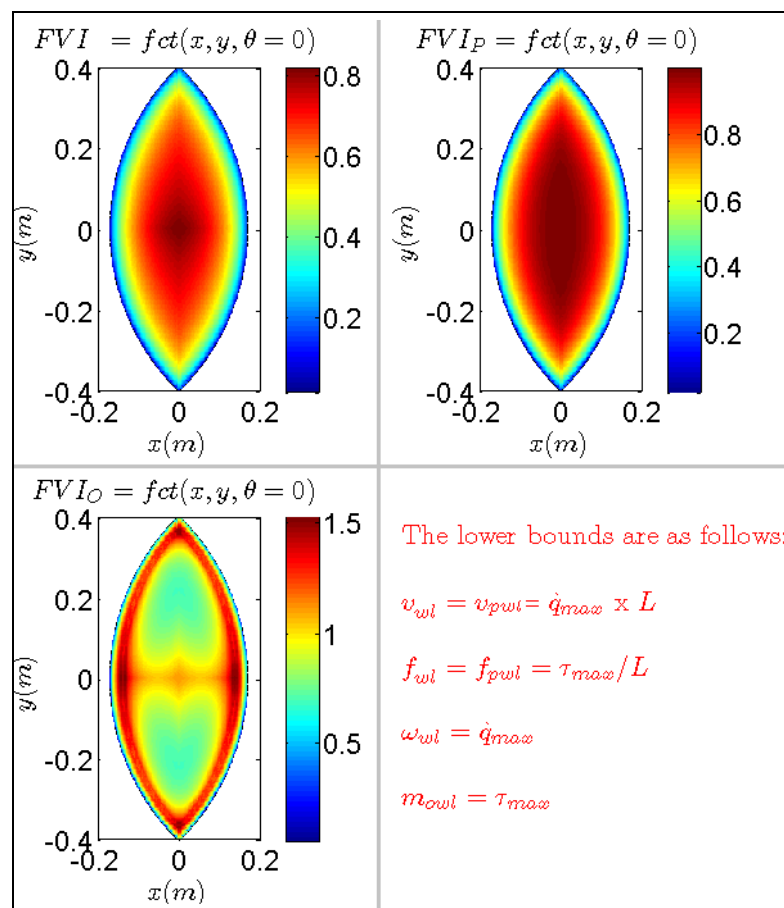

(a)

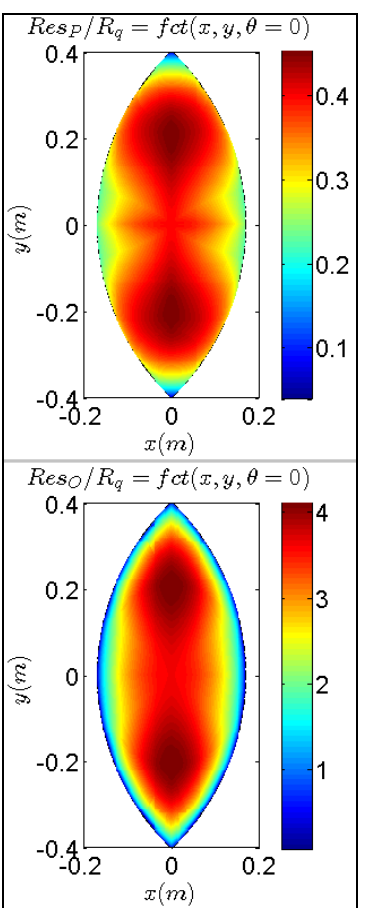

(b)

Figure 2. DUAL V Kinetostatic Evalution Based on FVI Indices (a) and Operational Resolutions (b): (a)- Homogenized FVI Index (Top Left), Translational FVI Index (Top Right), and Rotational FVI Index (Bottom Left), (b) - Translational and Rotational Resolutions' Ratios with Respect to Individual Actuator's Resolution (Top and Bottom Graphs Respectively). 
geometrical parameters for our study. One can derive the inverse Jacobian $\boldsymbol{J}_{\boldsymbol{m}}$ classically by deriving the inverse geometric model and differentiating it with respect to endeffector's pose; we will not detail this here for compactness reasons (the reader may refer to [22] for details).

Note that all the actuators are identical and the lower bounds for isotropic speeds, forces and moments are as follows:

$$
\left\{\begin{array}{l}
v_{w l}=v_{p w l}=\dot{q}_{\max } \times L \\
f_{w l}=f_{p w l}=\frac{\tau_{\max }}{L} \\
\omega_{w l}=\dot{q}_{\max }, \quad m_{w l}=\tau_{\max }
\end{array}\right.
$$

In Fig. 2(a), the three FVI indices (homogenized, translational and rotational indices) are presented as function of $x$ and $y$, while fixing $\theta=0^{\circ}$. In Fig. 2(b), we depict the operational translational and rotational resolutions' ratios with respect to the individual actuator's resolution $R_{q}$ (as all actuators are identical).

\section{CONCLUSIONS AND PERSPECTIVES}

In this article, we have exposed the issue related to performance evaluation in the case of mixed dofs robots, discussed some of literature works in this scope, and finally introduced our point of view and provided a relevant way to tackle the issue. Our approach has been based on kinetostatic performance measure recently suggested in [9-10] and on the translational and rotational operational resolutions. These measures provide concrete, clear and comprehensible physical significances that are undeniably useful for a robot designer. Moreover, this approach and these indices do not lose any of their significances in the case of redundant robots. In fact, they can be used on general robot whether serial, parallel, actuatedly or kinematically redundant, with mixed or homogeneous dofs, and with same or different types of actuators...To demonstrate this methodology, we have presented a case study on the actuatedly redundant parallel manipulator DUAL V with 3 dofs (2T-1R) planar motion.

Regarding future work, the methodology presented here will be further extended and developed to allow simultaneous assessment of isotropic translational and rotational capacities in kinetostatics and dynamics for a general robot, including cable-driven parallel robots. These studies will be the subject of future publications.

\section{REFERENCES}

[1] J. K. SALISBURY and J. J. CRAIG, "Articulated hands: force control and kinematic issues", International Journal of Robotics Research, Vol. 1, No. 1, pp. 4-17, 1982.

[2] T. YOSHIKAWA, "Manipulability of robotic mechanisms", International Journal of Robotics Research, Vol. 4, No. 2, pp. 3-9, 1985.

[3] R. STOUGHTON and T. KOKKINIS, "Some properties of a new kinematic structure for robot manipulators", Proc. 13th ASME Design Automation Conference, 73-79, Boston, MA (1987).

[4] F. XIE, X.-J. LIU, and J. WANG, "Performance Evaluation of Redundant Parallel Manipulators Assimilating Motion/Force
Transmissibility", International journal of Advanced Robotic Systems, Vol. 8, No. 5, pp. 113-124, 2011.

[5] C. CHEN, J. ANGELES, "Generalized transmission index and transmission quality for spatial linkages", Mechanism and Machine Theory, Vol. 42, pp. 1225-1237, 2007.

[6] J. WANG, C. WU, X.-J. LIU, "Performance evaluation of parallel manipulators: Motion/force transmissibility and its index", Mechanism and Machine Theory, Vol. 45, pp. 1462-1476, 2010.

[7] S. KRUT, O. COMPANY and F. PIERROT, "Velocity Performance Indexes for Parallel Mechanisms with Actuation Redundancy," in Proc. of 2002 Workshop on Fundamental Issues and Future Research Directions for Parallel Mechanisms and Manipulators, pp. 46-56, Quebec City, Quebec, Canada, October 3-4, 2002

[8] S. KRUT, Ph.D Thesis: "Contribution à l'étude des robots parallèles légers, 3T-1R et 3T-2R, à forts débattements angulaires », Université Montpellier II, Novembre 13, 2003.

[9] S. SHAYYA, S. KRUT, O. COMPANY, C. BARADAT and F. PIERROT, "A Novel (3T-1R) Redundant Parallel Mechanism with Large Operational Workspace and Rotational Capability", in Proc. of IEEE/RSJ International Conference on Intelligent Robots and Systems, IROS 2013, pp. 436-443, Tokyo, Japan, November 3-8, 2013.

[10] S. SHAYYA, S. KRUT, O. COMPANY, C. BARADAT and F. PIERROT, "A Novel (3T-2R) Parallel Mechanism with Large Operational Workspace and Rotational Capability", accepted paper to appear in Proceedings of 2014 IEEE International Conference on Robotics and Automation (ICRA 2014), Hong Kong, China, May 31 June 7, 2014.

[11] F. C. PARK, and J. W. KIM, "Manipulability of closed kinematic chains", ASME J. Mechanical Design. Vol.120. No.4. 1998.

[12] C. M. GOSSELIN, "The optimum design of robotic manipulators using dexterity indices", Robotics and Autonomous Systems, Vol. 9, No. 4, pp. 213-226, 1992.

[13] S. G. KIM, and J. RYU, "New dimensionally homogeneous Jacobian matrix formulation by three end-effector points for optimal design of parallel manipulators", IEEE Transactions on Robotics and Automation, Vol. 19, No. 4, pp. 731-737, 2003.

[14] S. G. KIM and J. RYU, "Force transmission analyses with dimensionally homogeneous jacobian matrices for parallel manipulators," KSME International Journal, Vol. 18, No. 5, pp. 780 788, 2004.

[15] I. MANSOURI and M. OUALI, "A new homogeneous manipulability measure of robot manipulators, based on power concept", Mechatronics, Vol. 19, No. 6, pp. 927-944, 2009.

[16] I. MANSOURI and M. OUALI, "The power manipulability-a new homogeneous performance index of robot manipulators", Robotics and Computer-Integrated Manufacturing, Vol. 27, No. 2, pp. 434449, 2011.

[17] J. P. MERLET, "Jacobian, manipulability, condition number, and accuracy of parallel robots," Journal of Mechanical Design, Transactions of the ASME, vol. 128, no. 1, pp. 199-206, 2006.

[18] G. BOSCHETTI, R. ROSA, and A. TREVISANI, "Parallel Robot Translational Performance Evaluation through Direction-Selective Index (DSI)", Journal of Robotics Volume 2011, Article ID 129506, 14 pages.

[19] O. MA, and J. ANGELES, "Optimum Architecture Design of Platform Manipulators", Proc. IEEE Int. Conf. Advanced Robotics, 1991.

[20] L. STOCCO, S. E. SALCUDEAN and F. SASSANI, "Matrix Normalization for Optimal Robot Design", in Proc. of IEEE ICRA, Leuven, Belgium, May 16-21, 1998.

[21] D. CHABLAT and P. WENGER, "Architecture Optimization of a 3DOF Parallel Mechanism for Machining Applications, the Orthoglide", IEEE Transactions On Robotics and Automation, Vol. 19/3, pp. 403-410, Juin, 2003.

[22] V. VAN DER WIJK, S. KRUT, F. PIERROT and J. L. HERDER, "Design and experimental evaluation of a dynamically balanced redundant planar 4-RRR parallel manipulator", The International Journal of Robotics Research, Vol. 32 , No. 6, pp. 744-759, May 2013. 